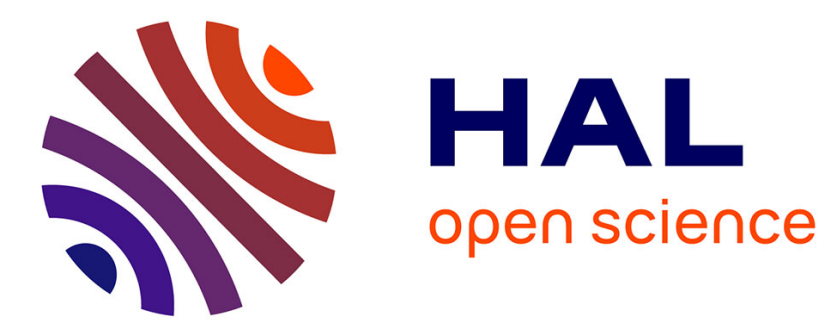

\title{
Séparation isotopique par passage du courant dans un métal fondu \\ P.-G. de Gennes
}

\section{To cite this version:}

P.-G. de Gennes. Séparation isotopique par passage du courant dans un métal fondu. J. Phys.

Radium, 1956, 17 (4), pp.343-346. 10.1051/jphysrad:01956001704034300 . jpa-00235376

\section{HAL Id: jpa-00235376 https://hal.science/jpa-00235376}

Submitted on 1 Jan 1956

HAL is a multi-disciplinary open access archive for the deposit and dissemination of scientific research documents, whether they are published or not. The documents may come from teaching and research institutions in France or abroad, or from public or private research centers.
L'archive ouverte pluridisciplinaire HAL, est destinée au dépôt et à la diffusion de documents scientifiques de niveau recherche, publiés ou non, émanant des établissements d'enseignement et de recherche français ou étrangers, des laboratoires publics ou privés. 


\title{
SÉPARATION ISOTOPIQUE PAR PASSAGE DU COURANT DANS UN MÉTAL FONDU
}

\author{
Par Pierre G. de GenNeS, \\ Centre d'Etudes Nucléaires du C. E. A., Saclay.
}

\begin{abstract}
Sommaire. - L'analyse des chocs ions-électrons dans un métal fondu comprènant 2 isotopes montre que la section efficace de l'isotope léger est supérieure à celle de l'isotope lourd ; en présence du courant, le premier est donc entraîné par les électrons. Le calcul quantitatif de la ségrégation qui en résulte suppose connus le nombre d'électrons libres dans le métal liquide, la relation entre coefficients de diffusion et de viscosité, et la fréquence moyenne d'oscillation d'un ion, mais il ne fait pas intervenir la masse électronique. Le résultat calculé est environ 2 fois plus faible que ne l'indique la mesure de Nieff et Roth sur le gallium.
\end{abstract}

1. Introduction. - Un courant continu (intensité typique 100 ampères $/ \mathrm{mm}^{2}$ ) passe pendant plusieurs jours dans un métal liquide comportant deux isotopes. L'isotope léger se concentre du côté anodique. L'effet a été observé en particulier sur le mercure [1], le gallium [2], etc...

Le signe de l'effet a conduit à envisager pour son interprétation les interactions ions-électrons [3]. Comme dans le traitement standard de la conductivité des métaux solides, nous analyserons ces interactions en termes de chocs ou de probabilités de transitions. Ceci nécessite quelques remarques.

On ne dispose que d'une description qualitative du mouvement " non perturbé » des électrons dans un métal liquide : le nuage électronique s'ajuste en $\sim 10^{-17}$ secondes sur le mouvement des ions (périodes typiques $10^{-13}$ secondes). Au potentiel de Hartree qui en résulte correspondent des niveaux instantanés d'électrons dans un champ de force non périodique (toutes les bandes ont une queue vers les hautes énergies). Au cours du mouvement des ions, ces niveaux sont déformés et les électrons d'énergie voisine du niveau de Fermi font des transitions entre eux; ils peuvent ainsi céder de l'impulsion aux ions.

On décrira ici les ions comme des atomes d'Einstein, oscillant dans un cage de voisins, sans corrélation entre eux : aux températures envisagées, ce modèle est satisfaisant (4). L'approximation centrale consiste à admettre que le potentiel perturbateur est proportionnel à l'amplitude du déplacement de l'ion par rapport à sa position moyenne dans la cage: Cette approximation est parfaitement justifiée pour un métal solide ; il est difficile d'évaluer l'erreur qu'elle implique ici. Une autre approximation consistera à admettre l'existence d'un temps moyen de parcours des électrons bien défini. Elle peut introduire une erreur numérique notable.

2. Mouvement d'entraînement des ions. 1. ÉQUation DE Mouvement. - Soit un métal à deux isotopes, 1 et 2 et posons : $p_{\mathrm{i}}$ : impulsion moyenne (d'entraînement) de l'espèce $\mathrm{i}$ dans le système du centre de masse $\mathrm{du}$ liquide.

( $p_{i}$ est déterminé par une moyenne sur - des temps $\sim 10^{-13}$ sec.)

$E$ : champ électrique.

$e$ : charge élémentaire.

$Z$ : nombre de charges par ion

$$
\frac{\mathrm{d} p_{\mathrm{i}}}{\mathrm{d} \Gamma}=\frac{-1}{\tau n i} p_{\mathrm{i}}+F+Z e E-\emptyset_{\mathrm{i}}
$$

$\frac{1}{\tau n i}$ définit la viscosité du métal pour l'isotope i.

$F$ désigne un gradient de pression (moyenne sur des temps $\gg 10^{-13}$ sec.)

$\varnothing_{i}$ une force moyenne due aux chocs électroniques (pendant une période d'oscillation il y a environ cent chocs électroniques : moyenne bien définie).

En régime stationnaire :

$$
\frac{\mathrm{d} p_{\mathbf{i}}}{\mathrm{d} t}=0 .
$$

2) Calcul des forces $\emptyset_{i}$. - On peut admettre que la diffusion des électrons par un ion est isotrope. En effet :

a) la longueur d'aćtion effective du potentiel coulombien de l'ion est $\sim 0,5 \widehat{A}$ pour le gallium; on peut considérer que le rayon d'action n'excède guère le rayon ionique $0,6 \widehat{\mathrm{A}}$, or la longueur d'onde moyenne des électrons est $\sim 3 \widehat{A}$;

b) la statistique tend à orienter l'impulsion finale de l'électron en direction opposée au courant moyen d'électrons, ce qui augmente l'impulsion moyenne cédée. Cet effet est très faible de l'ordre de $\frac{\mathrm{eE} \lambda}{\mathrm{KT}}$ ou $\lambda$ est le libre parcours moyen $\sim 10$ à $30 \AA$.

Soit $\tau_{\mathrm{e}}$ le temps moyen entre deux chocs d'un électrons avèc les ions. L'impulsion moyenne trans- 
férée à un ion par choc est en valeur absolue :

$$
e E \tau_{\mathrm{e}} \text {. }
$$

Le nombre de chocs par seconde sur l'espèce $\mathrm{i}$ sera désigné par $1 / \tau_{1}$.

$$
\emptyset_{i}=\frac{e E \tau_{e}}{\tau_{i}} .
$$

En comptant tous les chocs survenus en une seconde on trouve que :

$$
\cdot \frac{Z}{\tau_{e}}=\frac{\alpha_{1}}{\tau_{1}}+\frac{\alpha_{2}}{\tau_{2}}
$$

ou $\alpha_{1}$ et $\alpha_{2}$ désignent les pourcentages des deux isotopes $\alpha_{1}+\alpha_{2}=1$

posant

$$
\begin{gathered}
\frac{Z}{\tau_{\theta}}=\frac{1}{\tau} \\
\emptyset_{i}=Z e E \frac{\tau}{\tau_{i}}
\end{gathered}
$$

on vérifie que

$$
\alpha_{1} \varnothing_{i}+\alpha_{2} \varnothing_{2}=Z e E
$$

(toute l'impulsion électrique acquise par les élec. trons est cédée aux ions).

3) Courant de ségrégation. - En reportant II,6 dans II,2 on a pour le flux matière, défini par :

$$
P=\left(\alpha_{1} P_{1}+\alpha_{2} P_{2}\right) N
$$

$N$ : densité d'ions

$$
\begin{aligned}
& \frac{P}{N}=F\left(\tau_{n 1} \alpha_{1}+\tau_{n 2} \alpha_{2}\right) \\
& +Z e E\left[\tau_{n 1} \alpha_{1} \frac{\tau_{1}-\tau}{\tau_{1}}+\tau_{n 2} \alpha_{2} \frac{\tau_{2}-\tau}{\tau_{2}}\right]
\end{aligned}
$$

en vertu de (II,4) les deux termes qui figurent, dans le crochet sont de signes opposés ; nous montrerons qu'ils sont du premier ordre en $\frac{\Delta M}{M}$, variation relative de masse entre les deux isotopes. D'autre part $\tau_{n_{1}}$ et $\tau_{n_{2}}$ diffèrent aussi de termes du premier ordre en $\frac{\Delta M}{M}$.

En négligeant les termes du deuxième ordre et en posant :

$$
\begin{aligned}
& \tau_{n}=\alpha_{1} \tau_{n 1}+\alpha_{2} \tau_{n 2} \\
& \left.\left.\frac{P}{N} \sim F \tau_{n}+Z e E \tau_{n}\right] \frac{\tau_{1}-\tau}{\tau_{1}}+\frac{\tau_{2}-\tau}{\tau_{2}} \alpha_{1}\right] \\
& =F \tau_{n} .
\end{aligned}
$$

On écrira alors que le flux matière est nul

$$
\begin{aligned}
& P=0 \\
& F=0 \\
& p_{\mathrm{i}}=Z e E \frac{\tau_{\mathrm{i}}-\tau}{\tau} \times \tau
\end{aligned}
$$

le facteur $Z_{\mathrm{e}} \frac{\tau_{1}-\tau}{\tau_{\mathrm{j}}}$ est une charge effective. L'isotope qui a le plus petit $\tau_{i}$ (nombreux chocs avec les électrons) a une charge effective négative. L'autre isotope a une charge positive en vertu de (II, 1 t).

Définissons la vitesse de ségrégation par:

$$
U=\frac{1}{M}\left(p_{1}-p_{2}\right) \text {. }
$$

$M$ : masse moyenne des 2 types d'ions.

En négligeant toujours les termes en $\left(\frac{\Delta M}{M}\right)^{2}$

$$
U=\frac{Z e E}{M} \tau_{n} \tau\left(\frac{\tau_{2}-\tau_{1}}{\tau_{1} \tau_{2}}\right)
$$

4) Calgul des probabilités de tránsition. L'ion oscille dans sa cage avec une fréquence $\omega$ : le nombre de quanta d'oscillation est $n$. Il reçoit un électron d'impulsion $k$ et emet un électron d'impulsion $k^{\prime}$. Le déplacement de l'ion est $\vec{U}$, le potentiel perturbateur au point $\vec{r} \vec{W}(\vec{r}$. $) \vec{U}$ par hypothèse.

L'élément de matrice pour l'absorption d'un phonon est :

$\int \mathrm{d} \vec{r} \mathrm{~d} \vec{u} \Psi_{k}^{\times}(r) \Psi_{k^{\prime}}(r) W(\vec{r}) \vec{U} \Phi_{n}^{\times}(\vec{U})_{\Phi_{n-1}}(\vec{U})_{(\mathrm{II}, 18)}$ ou les $\Psi$ sont les fonctions électroniques non perturbées, supposées indépendantes de $\vec{U}$, les $\Phi_{n}$ sont le produit de 3 fonctions d'oscillateur harmonique.

L'élément de matrice se subdivise en trois termes de la forme :

$$
\int \mathrm{d} r \Psi_{k}^{\times} \Psi_{k^{\prime}} W(r)\left(\frac{\hbar^{\prime}}{M \bar{K}}\right)^{1 / 4}\left(\frac{n}{2}\right)^{1 / 2} .
$$

$\bar{K}$ : constante de force $\bar{K}=M \omega^{2}$.

$\bar{K}$ : indépendant de la masse de l'isotope.

On peut le résumer sous la forme :

$$
A_{k k^{\prime}} \times\left(\frac{n \hbar \omega}{2 \bar{K}}\right)^{1 / 2}
$$

ou $A_{K K^{\prime}}$ ne dépend que des paramètres électroniques.

De la même façon, l'élément de matrice pour l'émission est de la forme :

$$
A_{k k^{\prime}}\left[\frac{(n+1) \hbar \omega}{2 \bar{K}}\right]^{1 / 2}
$$

La probabilité totale de transition est : $\frac{1}{\tau_{1}}=\frac{2}{\hbar} \sum_{K K^{\prime}} \frac{\hbar \omega_{1}}{\bar{K}}\left|A_{k k^{\prime}}\right|^{2} n_{k}\left(1-n_{k^{\prime}}\right)\left[n \Omega+(n+1) \Omega_{+}\right]$

où $n_{k}, n_{k^{\prime}}$ sont les nombres d'occupation électroniques 
eft

$$
\Omega \quad=\frac{\sin \left(E_{k}-\cdots E_{k^{\prime}} \pm h \omega_{\mathrm{i}}\right) \mathrm{t} / h}{E_{k}-E_{k^{\prime}} \pm \hbar \omega_{\mathrm{i}}} .
$$

Les $\Omega$ ne peuvent être ici remplacés par $\delta\left(E_{k}-E_{k^{\prime}} \pm \hbar \omega_{i}\right)$ car le temps $t$ est inférieur à $\tau_{\mathbf{e}} \lesssim 3.10^{-13}$ secondes $;\left(\frac{\hbar}{\tau_{\mathrm{e}}} \sim 30 k T\right.$. $)$ on peut admettre au contraire que les $\Omega$ sont égales dans le domaine d'énergies qui nous intéresse (et égales en moyenne à $\frac{\tau_{\mathrm{e}}}{2 \hbar}+$ ).

Dans ces conditions :

$$
\begin{gathered}
\frac{1}{\tau_{1}}=\text { cte } \times\left(n+\frac{1}{2}\right) \hbar \omega_{i} \\
\frac{1}{\tau_{i}} \sim \frac{\hbar \omega_{i}}{e^{\hbar \omega_{i} / K T}-1}+\frac{1}{2} \hbar \omega_{i} \\
=K T+\frac{1}{12} \frac{\left(\hbar \omega_{i}\right)^{2}}{(K T)}+\ldots \text { pour } K T \gg \hbar \omega
\end{gathered}
$$

(ce qui est bien vérifié ici).

D'où la variation relative de $\frac{1}{\tau_{i}}$ entre les deux isotopes.

$$
\frac{\Delta\left(\frac{1}{\tau}\right)}{\frac{1}{\tau}}=\frac{\Delta \omega}{\omega} \frac{1}{6}\left(\frac{\hbar \omega}{K T}\right)^{2}=\frac{1}{12} \frac{\Delta M}{M}\left(\frac{\hbar \omega}{K T}\right)^{2} .
$$

Le sens physique de ce résultat est le suivant : l'isotope léger est un peu plus " délocalisé » que l'isotope lourd (ou, si l'on veut, son oscillation du point 0 est supérieure) : son amplitude de vibration étant plus grande, il perturbe plus le mouvement des électrons, subit plus de chocs (et est entraîné par eux).

Dans la suite nous poserons :

$$
\overline{(\hbar \omega)^{2}}=K^{2} \Theta^{2} \text {. }
$$

5) Calcul de $\tau_{n}$. - Si l'on admet la même énergie d'activation pour les processus de diffusion et de viscosité, on a la relation (5)

$$
\tau_{n}=C \frac{M D}{K T}
$$

ou $D$ est le coefficient de self diffusion, $C$ un facteur numérique de l'ordre de grandeur de 1 , pour le gallium à $400^{\circ} \mathrm{K}$, on constate que $C=1$ est une bonne approximation : on obtient en effet pour $\tau_{n}$ des valeurs comparables $\left(\tau_{n} \simeq 6.10^{-14}\right)$, en le déduisant de la formule précédente avec $C=1$, ou de la formule:

$$
\tau_{n}=\frac{M}{6 \pi \eta a}
$$

avec $\eta=2.10^{-2}$ poises

$$
a \sim \text { rayon ionique } 0,610^{-8} \mathrm{~cm} \text {. }
$$

D'où la formule finale, en utilisant (II, 17), (II, 23) et (II, 25).

$$
\frac{U}{D}=\frac{Z e E}{12 K T} \times C \frac{\Theta^{2}}{T^{2}} \frac{\Delta M}{M} .
$$

6) ÉTude du RÉGIMe de diffusion. - Soient $\alpha_{1}+\varepsilon, \alpha_{2}-\varepsilon$ les pourcentages isotopiques.

Les variations $\varepsilon$ sont petites $\left(\varepsilon<\frac{1}{20}\right.$ dans l'expérience citée plus loin sur le gallium).

Les courants sont :

$$
\begin{aligned}
& j_{1}=-D_{1} \frac{\partial \varepsilon}{\partial \varkappa}+\left(\alpha_{1}+\varepsilon\right) \frac{P_{1}}{M_{1}} \\
& j_{2}=+D_{2} \frac{\partial \varepsilon}{\partial \varkappa}+\left(\alpha_{2}-\varepsilon\right) \frac{P_{2}}{M_{2}}
\end{aligned}
$$

on doit négliger les termes en $\varepsilon \frac{\Delta M}{M}$

posant

$$
\frac{P_{\mathrm{i}}}{M}=V_{\mathrm{i}}
$$

$$
j_{1} \simeq-D \frac{\partial \varepsilon}{\partial x}+\alpha_{1} V_{1}
$$$$
j_{2} \simeq D \frac{\partial \varepsilon}{\partial x}+\alpha_{2} V_{2}
$$

$$
j_{1}+j_{2} \simeq 0
$$

la conservation des particules donne

$$
\begin{gathered}
\operatorname{div} j_{1}+\frac{\partial \varepsilon}{\partial t}=0 \\
-D \frac{\partial^{2} \varepsilon}{\partial x^{2}}+\frac{\partial \varepsilon}{\partial t}=0
\end{gathered}
$$

avec pour "conditions aux limites (longueur du capillaire : $2 l$ )

$$
\begin{aligned}
& \left(\frac{\partial \varepsilon}{\partial x}\right)_{x= \pm l}=\frac{\alpha_{1} V_{1}}{D} \\
& \cdot[\varepsilon(x)]_{t=0}=0 .
\end{aligned}
$$

La solution est

$$
\begin{gathered}
\varepsilon=\frac{\alpha_{1} V_{1} x}{D}-\sum_{n>0} \frac{16 \alpha_{1} V_{1} l}{n^{3} D} \frac{1}{\frac{\sin (2 n+1)^{2}}{2 l}} \\
\theta_{n}=\frac{1}{(2 n+1)^{2}} \frac{(2 l)^{2}}{\pi^{2} D}
\end{gathered}
$$

a) à l'équilibre

$$
\frac{\partial \varepsilon}{\partial x}=\alpha_{1} \varkappa_{2} \frac{U}{D}
$$

en posant

$$
\begin{aligned}
& K_{1}=\frac{\alpha_{1}-\varepsilon}{\alpha_{2}+\varepsilon} \\
& \frac{1}{K_{1}} \frac{\partial K_{1}}{0 x}=\frac{U}{D}
\end{aligned}
$$

(II, 35 
b) on peut déduire une valeur approchée $D$ de l'approche vers l'équilibre ; pour une expérience suffisamment longue, seul le premier terme de $\Sigma$ dans (II, 32) intervient :

$$
\varepsilon(l, t)=\alpha_{1} \alpha_{2} \frac{U l}{D}\left(1-\frac{16}{\pi^{3}} \mathrm{e}^{-t / \theta_{0}}\right)
$$

on connaît $\alpha_{1} \alpha_{2} \frac{U}{D}$ par la pente de la courbe $\varepsilon(x)$ aux points \pm 1 , mêmè si l'équilibre n'est pas atteint connaissant $\varepsilon(l t)$ on peut donc déduire $\theta_{1}$ d'où $D$.

3. Application numérique au gallium et conclusions. - En utilisant les données numériques de Nieff et Roth [2] on trouve très approximavement

$$
\Xi=3.10^{-5} \mathrm{~cm} 2 / \mathrm{s} .
$$

On admet :

a) une température du métal de $400^{\circ} \mathrm{K}$ environ (déduite de la conductivité thermique du capillaire) ;

b) une température $\Theta$ d'environ $T / 3$ (la température d'Einstein du gallium solide est d'environ $100^{\circ} \mathrm{K}$, on est conduit à penser que $\theta$ est plus élevé dans le métal liquide plus compact);

c) un nombre d'électrons porteurs du courant $Z=3$ (en fait, compris entre 1 et 3 , mais certainement plus élevé pour le liquide que pour Ie solide).

On trouve $U / D \sim \frac{1}{200} \mathrm{~cm}^{-1}$ pour l'expérience citée, alors que le résultat expérimental est environ $\frac{1}{80} \mathrm{~cm}^{-1}$.

L'origine de ce désaccord numérique est liée :

a) au choix de la forme du potentiel perturbateur ;

b) à l'usage d'un temps de libre parcours électronique ; c) au caractère incertain de la relation entre diffusion et viscosité ;

d) au caractère anharmonique des vibrations. La variation thermique de $\frac{U}{D}$ ne peut être prédite par (II, 26) sans connaissance plus détaillée des variations de $Z$ et $C$. On peut penser que $Z$ augmente avec la température (dans l'évaluation numérique nous avons admis $Z=Z_{\max }=3$ ). La variation de $C$ dépend de la différence des énergies d'activation de diffusion et de viscosité, et peut être rapide, de signe quelconque.

La comparaison de l'effet entre les différents métaux conduit à envisager le facteur $Z \frac{\Theta^{2}}{T_{\mathbf{F}^{3}}}$ ou $T_{\mathbf{F}}=$ température de fusion.

Ce facteur est particulièrement élevé dans les groupes des alcalins, du gallium, du zinc. (Ici encore, une évaluation de $Z$ à l'état liquide est nécessaire pour une comparaison précise.)

On peut se demander enfin si l'effet est observable en dessous de la fusion. Au premier abord, (II, 25) restant valable, la formule (II, 26) paraît applicable. Cette conclusion est incorrecte. En effet, notre description en atomes d'Einstein perd beaucoup de sa validité, l'électron n'échange pas son impulsion avec un ion, mais avec un groupe d'ions voisins, et l'entraînement sélectif devient beaucoup plus faible.

L'existence d'un effet inverse (apparition d'une différence de potentiel en présence d'un gradient de concentration) a été suggérée par $M$. Yvon et envisagée indépendamment par $M$. Nieff. Il peut être calculé par la relation d'Onsager et parât trop faible pour être mesurable.

Je tiens à remercier M. A. Herpin qui m'a suggéré ce problème, ainsi que le $\mathrm{Pr}^{\mathrm{E}} \mathrm{E}$. Bauer avec qui nous avons longuement discuté à ce sujet. Je remercie également M. P. Aigrain et tout particulièrement MM. Nieff et Goldmann qui m'ont fourni les résultats expérimentaux indispensables.

Manuscrit reçu le $1^{\text {er }}$ février 1956.

\section{BIBLIOGRAPHIE}

[1] Haeffner, Nature, 1953, 172, 775.

[2] Nieff et Roth, $C . R$. Acad. Sc., 5 juillet 1954.

13] Kцемм, Naturforsch., 1954, 9 a, 1031.
[4] Motт et Jones, Theory of metals and alloys, Oxford. [5] Hirschfelder, Curtiss, Bird, Molecular theory of gases and liquids, 625 . 